This document is the Accepted Manuscript version of a Published Work that appeared in final form in Journal of the American Chemical Society, copyright $\odot$ American Chemical Society after peer review and technical editing by publisher. To access the final edited and published work see Smith, $B$. D., \& Liu, J. (2010). Assembly of DNA-Functionalized Nanoparticles in Alcoholic Solvents Reveals Opposite Thermodynamic and Kinetic Trends for DNA Hybridization. Journal of the American Chemical Society, 132(18), 6300-6301.

https://doi.org/10.1021/ja101973g

\title{
Assembly of DNA-Functionalized Nanoparticles in Alcoholic Solvents Reveals Opposite Thermodynamic and Kinetic Trends for DNA Hybridization
}

\author{
Brendan D. Smith and Juewen Liu* \\ Department of Chemistry, Waterloo Institute for Nanotechnology, University of Waterloo, 200 University Avenue West, \\ Waterloo, Ontario, N2L 3G1, Canada
}

RECEIVED DATE (Received March 8, 2010); liujw@uwaterloo.ca

In the past twenty years, DNA has been a key component in bionanotechnology, ${ }^{1}$ which can be attributed to the development of DNA chemical synthesis, the availability of a wide variety of enzymes for DNA manipulation, and the high fidelity and programmable DNA base pairing interactions. So far, majority of the experiments involving DNA have been performed in aqueous solutions, as it is common knowledge that organic solvents such as ethanol can denature and precipitate DNA, which is actually a standard operation in DNA extraction. This may have led to the perception that DNA hybridization is slower and less stable in organic solvents, which in turn could explain the lack of related literature reports. To the best of our knowledge, all studies on the effects of organic solvents have focused on the thermodynamic properties such as DNA melting temperature $\left(\mathrm{T}_{\mathrm{m}}\right)$ and the B-to-A form transition for long DNA with kilobase pairs, ${ }^{2}$ but not on the hybridization kinetics of short synthetic DNA. Our interests in this particular topic are for the following reasons. First, we wish to extend our understanding to kinetic properties. Second, we may extend the application of DNA-based technologies in areas which require organic solvents. Finally, organic solvents may improve the performance of DNA-based materials and sensors, and very interestingly, we found that DNA hybridization was faster in many alcohol/water co-solvents than in water, even though with a lower $\mathrm{T}_{\mathrm{m}}$. We herein communicate the kinetic and thermodynamic effects of alcohols on DNA hybridization using DNA-functionalized gold nanoparticles (AuNPs) as a model system.

Since 1996, DNA-directed assembly has become one of the corner stones in bionanotechnology. ${ }^{3}$ Many novel materials have been prepared with unique properties and important applications. ${ }^{4}$ We chose to use a standard two-particle-and-one-linker system as shown in Figure 1B. Both AuNPs were functionalized with thiolmodified DNA and can be assembled by a linker DNA, resulting in a red-to-purple color change in an aqueous buffer., 5

Before testing the effects of organic solvents on DNA-directed assembly, the property of AuNPs in such solvents was first studied. Citrate capped $13 \mathrm{~nm}$ AuNPs were dispersed in either 40\% (v/v) ethanol or water with varying salt concentrations. As shown in Figure 2A-B, AuNPs are less stable in ethanol since the color change to blue occurred with $>20 \mathrm{mM} \mathrm{NaCl}$; in water the color change occurred with $>50 \mathrm{mM} \mathrm{NaCl}$, which can be

attributed to a higher dielectric constant of water. Such a red-toblue color change is indicative of colloidal AuNP aggregation, and for citrate capped AuNPs, the salt induced aggregation is irreversible. When AuNPs were functionalized with the thiolmodified DNA, their stability is significantly improved. For example, color change was not observed until $200 \mathrm{mM} \mathrm{NaCl}$ was added in $40 \%$ ethanol (Figure 2C); while in water, even $300 \mathrm{mM} \mathrm{NaCl}$ did not induce a color change (Figure 2D). In addition, the aggregation is reversible for DNA protected AuNPs. The resulting blue colored samples (e.g. the ones in Figure 2C) can change color back to red upon heating (data not shown), suggesting that the color changes should be related to DNA-mediated reversible interactions. It needs to be pointed out that no linker DNA was added in the previous steps and therefore if DNA hybridization was responsible for the observed color change, it must be due to self-hybridization of DNA on AuNPs (Figure 1A). ${ }^{7}$

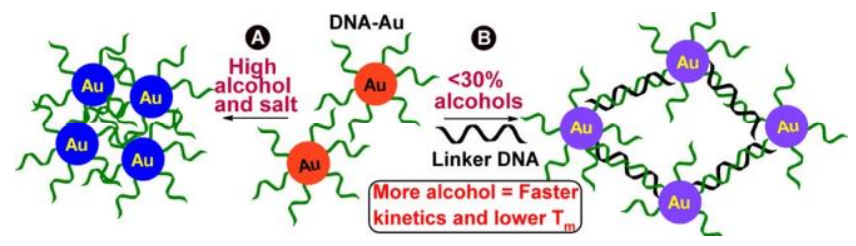

Figure 1. Schematic representation of DNA-functionalized AuNP selfaggregation (A) and DNA-directed assembly of AuNPs (B) in organic solvents with a red-to-blue or purple color change.

We further varied the percentages of three alcohols while keeping the ionic strength at $50 \mathrm{mM} \mathrm{NaCl}$. In the case of methanol (Figure $2 \mathrm{E}$ ), the color immediately changed to blue (indicating aggregation) when $>80 \%$ methanol was present. For ethanol and isopropanol, the color change was also instantaneous when $>60 \%$ of the alcohols were added. However, none of these blue-colored aggregates changed color back to red even when heated in a boiling water bath. We achieved the red color only by centrifugation, removal of the organic solvents, and adding water (the last tubes in Figure 2E-G), suggesting that the particles were still reversibly aggregated. The failure of heat induced color change may be explained by a drastic increase in the melting temperature $\left(\mathrm{T}_{\mathrm{m}}\right)$ of DNA and strong electrostatic interactions in concentrated alcohols. ${ }^{2 \mathrm{c}}$ On the other hand, the boiling point (bp) of the co-solvents is lower than that of water (e.g. $b p=78 \mathrm{CC}$ for pure ethanol). Interestingly, salt plays a critical role here. If very low $\mathrm{NaCl}$ (e.g. $8 \mathrm{CM}$ ) was present, the AuNPs were still red even in $98 \%$ of the alcohols (see Supporting Information). This is consistent with the notion that salt is needed for alcohol-induced DNA precipitation. Although no linker DNA was added, the fact that AuNPs can immediately and reversibly change color by alcohol suggest that DNA was quickly brought close to each other under such conditions, making it possible for fast hybridization.

To study the effect of organic solvents on specific DNA hybridization (based on Watson-Crick base paring), a linker DNA was added to the AuNPs in $0-40 \%$ methanol with $50 \mathrm{mM} \mathrm{NaCl}$. Higher alcohol contents were not included so that the observed color change can only be attributed to the linker DNA induced assembly. After $1 \mathrm{hr}$, the samples appeared to be progressively more purplish with higher methanol content (Figure 3A), suggesting a faster DNA hybridization. To quantitatively study the color change, UV-visible spectroscopy was used. As shown in Figure 3B, dispersed AuNPs have a characteristic extinction peak at $520 \mathrm{~nm}$. Upon aggregation, the $520 \mathrm{~nm}$ peak decreases in intensity and the $650 \mathrm{~nm}$ region extinction increases. Therefore, the extinction ratio of 650 over $520 \mathrm{~nm}$ was used to quantify the color and assembly state of the system. Upon assembly, this ratio should increase as the 
color changes to purple. As shown in Figure 3C, the color gradually changed in $1 \mathrm{hr}$ in the presence of the linker (with $30 \%$ methanol); while in the absence of the linker, the extinction ratio remained low. We next systematically compared the extinction ratio at $1 \mathrm{hr}$ after addition of the linker DNA as a function of alcohol concentration. The higher the alcohol content, the higher the extinction ratio or the faster the assembly (Figure 3D-F, blue dots). Again, such increased color change rate can only be attributed to specific AuNP aggregation, since in the absence of the linker DNA, there was no color change (red dots). If even higher percentages of the three alcohols were used, the samples without the linker started to turn blue and nonspecific interactions may start to dominate (see Supporting Information). These experiments demonstrate that in contrary to the traditional perception of DNA denaturation in organic solvents, we observed significantly faster hybridization kinetics if the volume fraction of alcohols was less than $\sim 30 \%$.

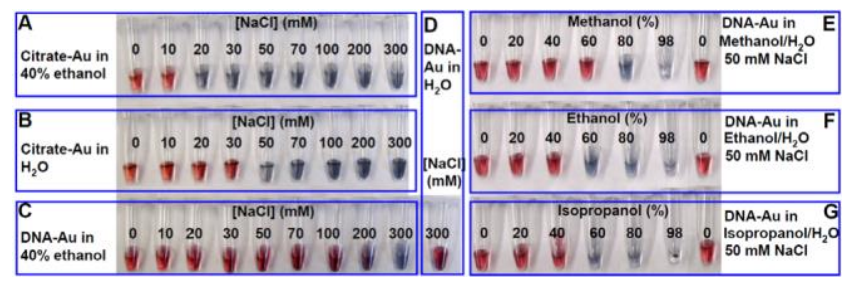

Figure 2. The effect of alcohols and salt on the stability of citrate (A-B) and thiol-modified DNA protected (C-G) AuNPs. A blue color indicates aggregated AuNPs. The last tubes in (E-G) were obtained by centrifugation of the tube next to them ( $98 \%$ alcohols) and then adding an aqueous buffer (50 mM NaCl, $10 \mathrm{mM}$ HEPES, pH 7.6).
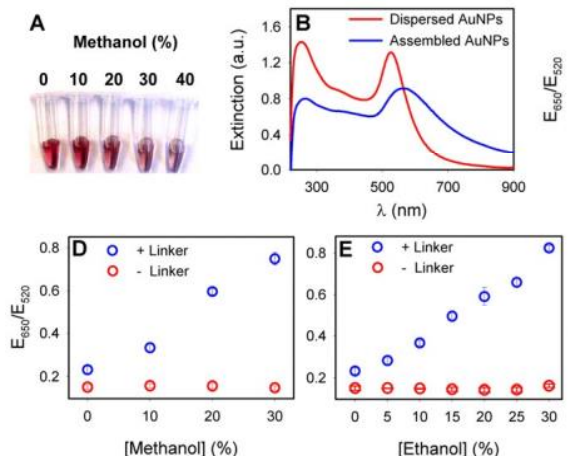

[Ethanol] (\%)

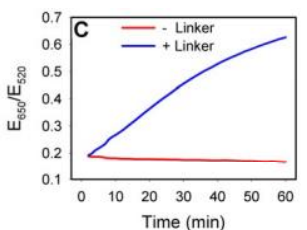

Time (min)

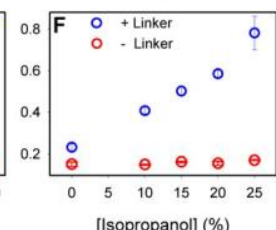

Figure 3. (A) A photograph of DNA-directed assembly of AuNPs in varying percentages of methanol. (B) UV-vis spectra of dispersed and assembled AuNPs. (C) The kinetics of color change of AuNPs induced by DNA-directed assembly in 30\% methanol. The extinction ratio of AuNPs after assembling for $1 \mathrm{hr}$ in the presence of varying concentrations of methanol (D), ethanol (E), and isopropanol (F).

To test the thermodynamic property of DNA-assembled AuNPs in organic solvents, we next studied their melting behavior by monitoring the extinction at $260 \mathrm{~nm}$, which increases sharply upon melting. As shown in Figure 4, a higher alcohol percentage resulted in a lower $\mathrm{T}_{\mathrm{m}}$ for all three alcohols, which is consistent with previous literature reports on the DNA denaturing effects of these solvents. ${ }^{2}$ In all of the melting curves, a sharp melting transition was observed, suggesting that the multivalent and cooperative DNA binding among AuNPs was still valid in the organic solvents. ${ }^{8}$ Given the decreased $T_{m}$, the cause for the increased hybridization kinetics is certainly not thermodynamic.

One explanation is that the water activity is decreased in the presence of the alcohols. ${ }^{9}$ Since DNA tends to be hydrated, its effective concentration is increased, leading to faster hybridization.
The decreased solvent dielectric constant may also contribute to the faster hybridization.

To test whether the observed effect is unique to our AuNP system, DNA hybridization experiments were performed in two molecular beacon systems with varying DNA sequences and lengths (no AuNPs involved). In the presence of complementary DNA, DNA hybridization results in fluorescence quenching or dequenching (see Supporting Information). The DNAs were allowed to hybridize in an aqueous buffer and in $25 \%$ ethanol. Indeed the hybridization in alcohols was three to four times more rapid as indicated by the fluorescence change. As a control, if noncomplementary DNAs were used, no time-dependent fluorescence change was observed. These experiments suggest that the faster hybridization in alcohols is general.

In summary, we have demonstrated that DNA hybridization is faster in alcohols, even though the $\mathrm{T}_{\mathrm{m}}$ is decreased. Therefore, there is an opposite kinetic and thermodynamic trend for DNA hybridization in diluted alcohols. This discovery extends not only the application of DNA bionanotechnology to organic solvents with improved performance but also our understanding on the DNA biophysics.

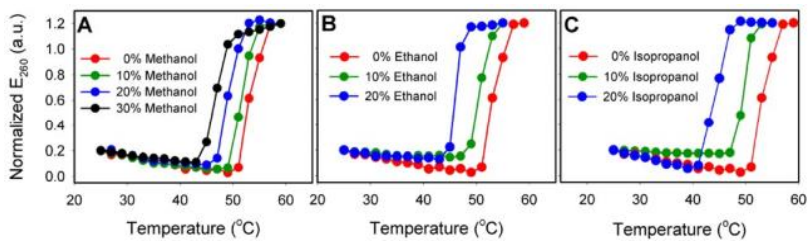

Figure 4. Melting curves of DNA-linked AuNPs in various percentages of methanol (A), ethanol (B), and isopropanol (C). All the samples contain 50 $\mathrm{mM} \mathrm{NaCl}$ and $20 \mathrm{mM}$ HEPES, $\mathrm{pH}$ 7.6.

ACKNOWLEDGMENT. This work is supported by the University of Waterloo and the Natural Sciences and Engineering Research Council (NSERC) of Canada (Discovery Grant and Undergraduate Fellowship).

Supporting Information Available: Materials and methods, additional data on AuNPs and fluorescence. This material is available free of charge via the Internet at http://pubs.acs.org.

\section{REFERENCES}

(1) (a) Storhoff, J. J.; Mirkin, C. A. Chem. Rev. 1999, 99, 1849-1862. (b) Seeman, N. C. Nature 2003, 421, 427-431. (c) Feldkamp, U.; Niemeyer, C. M. Angew. Chem., Int. Ed. 2006, 45, 1856-1876. (d) Aldaye, F. A.; Palmer, A. L.; Sleiman, H. F. Science 2008, 321, 1795-1799. (e) Liu, J.; Cao, Z.; Lu, Y Chem. Rev. 2009, 109, 1948-1998.

(2) (a) Herskovits, T.; Singer, S. J. Arch. Biochem. Biophys. 1961, 94, 99114 (b) Usatyi, A. F.; Shlyakhtenko, L. S. Biopolymers 1974, 13, 24352446. (c) Piskur, J.; Rupprecht, A. Febs Letters 1995, 375, 174-178. (d) Norberg, J.; Nilsson, L. Biophys. J. 1998, 74, 394-402. (e) Bonner, G.; Klibanov, A. M. Biotechnol.Bioeng. 2000, 68, 339-344.

(3) (a) Mirkin, C. A.; Letsinger, R. L.; Mucic, R. C.; Storhoff, J. J. Nature 1996, 382, 607-609. (b) Alivisatos, A. P.; Johnsson, K. P.; Peng, X.; Wilson, T. E.; Loweth, C. J.; Bruchez, M. P., Jr, et al. Nature 1996, 382 609-611.

(4) (a) Rosi, N. L.; Mirkin, C. A. Chem. Rev. 2005, 105, 1547-1562. (b) Rosi, N. L.; Giljohann, D. A.; Thaxton, C. S.; Lytton-Jean, A. K. R.; Han, M. S.; Mirkin, C. A. Science 2006, 312, 1027-1030. (c) Lu, Y.; Liu, J. Curr. Opin. Biotechnol. 2006, 17, 580-588. (d) Zhao, W.; Brook, M. A.; Li, Y. Chembiochem 2008, 9, 2363-2371.

(5) Elghanian, R.; Storhoff, J. J.; Mucic, R. C.; Letsinger, R. L.; Mirkin, C. A. Science 1997, 277, 1078-1080.

(6) Storhoff, J. J.; Elghanian, R.; Mucic, R. C.; Mirkin, C. A.; Letsinger, R. L. J. Am. Chem. Soc. 1998, 120, 1959-1964.

(7) Hurst, S. J.; Hill, H. D.; Mirkin, C. A. J. Am. Chem. Soc. 2008, 130, $12192-$ 12200 .

(8) Jin, R.; Wu, G.; Li, Z.; Mirkin, C. A.; Schatz, G. C. J. Am. Chem. Soc 2003, 125, 1643-1654.

(9) Hallsworth, J. E. J. Ferment. Bioeng. 1998, 85, 125-137. 